\title{
COPD: What the anaesthetist should know
}

\author{
ML Wong \\ Principal Specialist and Head:Division of Pulmonology, Chris Hani Baragwanath Academic Hospital and \\ School of Clinical Medicine, Faculty of Health Sciences, University of the Witwatersrand \\ Corresponding author, email: michelle.wong@wits.ac.za
}

Keywords: chronic obstructive pulmonary disease, COPD

\section{Introduction}

Chronic obstructive pulmonary disease (COPD) is a chronic, progressive, inflammatory disease affecting the airways and lung parenchyma. It is characterised by persistent airflow obstruction which may be partially reversible, and is caused by exposure of the lung to noxious particles or gases. ${ }^{1}$ Although preventable and treatable, once established, the disease course is punctuated by exacerbations. The consequences of COPD result in not just a disease of the respiratory system, but rather a systemic illness which affects multiple organs.

COPD is a common disease, yet frequently unrecognised and underdiagnosed. The WHO predicts it will become the $3^{\text {rd }}$ leading cause of death worldwide by $2030 .^{2}$

There has been a trend away from classifying COPD patients as having either emphysema ("pink puffers") or chronic bronchitis ("blue bloaters"). However, there is now mounting evidence that this may become clinically useful in stratifying patients with regard to prognosis and making treatment decisions.

More topically, there is an enhanced appreciation that some patients with COPD manifest with features of coexistent asthma. This entity has been labelled the asthma-COPD overlap syndrome (ACOS).

\section{Diagnosis}

The diagnosis of COPD hinges on three factors: (1) compatible symptoms in varying degrees (dyspnoea, chronic cough, chronic sputum production), (2) risk factors predisposing to the disease, and (3) post-bronchodilator spirometry demonstrating an obstructive lung function defect which may be partially reversible or irreversible. Tobacco smoking is the most common identifiable risk factor, but there are many other causes (e.g. previous pulmonary $\mathrm{TB}$, occupational exposures, etc.). In a recent prospective Canadian study, never smokers constituted $27 \%$ of COPD patients. ${ }^{3}$ This is likely to be even higher in South Africa where TB is endemic and exposure to biomass fuel more prevalent.

\section{Assessment of the COPD patient}

other than a clinical examination, the evaluation of the COPD patient should include detailing the severity of respiratory symptoms, quantifying the degree of airflow obstruction, assessing the risk of exacerbations and identifying comorbid illnesses. All these factors impact on quality of life, morbidity and mortality. Spirometry abnormalities alone provide an inadequate assessment of the disease and its pathophysiological consequences.

Symptomatology is comprehensively embodied by the COPD assessment test (CAT). This is an 8-question survey, where each symptom is scored from 1 to $5 .{ }^{4}$ A CAT score of $\geq 10$ is considered a high level of symptoms. A less comprehensive alternative is the modified MRC (mMRC) dyspnoea scale (see Table 1).

Spirometry shows evidence of airflow obstruction (FEV 1 FVC $<70 \%$ ). The severity of airflow obstruction is determined by the $\mathrm{FEV}_{1} \%$ predicted (see Table 2 ).

Table 1: Modified MRC dyspnoea scale

- Grade 0: breathless with strenuous exercise

- Grade 1: breathless when hurrying on the level or walking up a slight hill

- Grade 2: breathless when walking with people of the same age on the level, or need to stop for breath when walking at own pace on the level

- Grade 3: need to stop for breath when walking about $100 \mathrm{~m}$ or after a few minutes on the level

- Grade 4: too breathless to leave the house or breathless when dressing or undressing

Table 2: Gold classification of airflow obstruction

\begin{tabular}{lll}
\hline & \multicolumn{2}{c}{ FEV $_{\mathbf{1}} /$ FVC $<\mathbf{7 0} \%$} \\
\hline & Grade & Post-bronchodilator fev $_{\mathbf{1}}$ \\
\hline GOLD 1 & Mild & $\geq 80 \%$ predicted \\
GOLD 2 & Moderate & $50 \%$ to $<80 \%$ predicted \\
GOLD 3 & Severe & $30 \%$ to $<50 \%$ predicted \\
GOLD 4 & Very severe & $<30 \%$ predicted \\
\hline
\end{tabular}


Exacerbations of COPD (defined as worsening of respiratory symptoms beyond the usual day-to-day variation, and which necessitates a change in usual medication) have been shown to portend a worse prognosis. In a large prospective study, $45 \%$ of patients hospitalised for an exacerbation died within 4 years. ${ }^{5}$ It has also been established that there are different phenotypes of COPD, with frequent exacerbators ( $\geq 2$ episodes per year) suffering greater morbidity and mortality and worse quality of life. The number of exacerbations does not necessarily parallel the severity of airflow obstruction, although those with lower $\mathrm{FEV}_{1} \%$ predicted do tend to have more frequent exacerbations. ${ }^{6}$ Frequent exacerbations or one or more exacerbations per year requiring hospitalisation are markers for more rapid deterioration in lung function, poorer quality of life and increased morbidity and mortality. Exacerbations may be precipitated by many different factors, but the most common precipitant is a respiratory tract infection, either bacterial or viral.

Comorbid illnesses in COPD patients are important to identify. In fact, patients with COPD are more likely to die from comorbidities, particularly cardiovascular disease, than of pulmonary disease. ${ }^{7}$

In 2013, the Global Initiative for Obstructive Lung Disease (GOLD) introduced a more comprehensive approach to the assessment of the COPD patient, classifying patients into 4 categories: A, B, $C$ and $D$ (see Figure 1). This system, although flawed in certain areas, is designed to predict future risk in COPD patients and provides some guidance to the choice of drug therapy.

\section{Management}

COPD is an irreversible, but manageable disease. There are no disease-modifying drugs available. The only management options which have been shown to impact favourably on survival are smoking cessation and in those with end-stage disease complicated by respiratory failure, long-term oxygen therapy.

Pharmacological therapy is aimed at symptom relief, improvement in quality of life and exercise tolerance, and reduction of exacerbations. Drug options include bronchodilators ( $\beta_{2}$-agonists and/or antimuscarinic agents), corticosteroids, methylxanthines and phosphodiesterase-4 inhibitors.

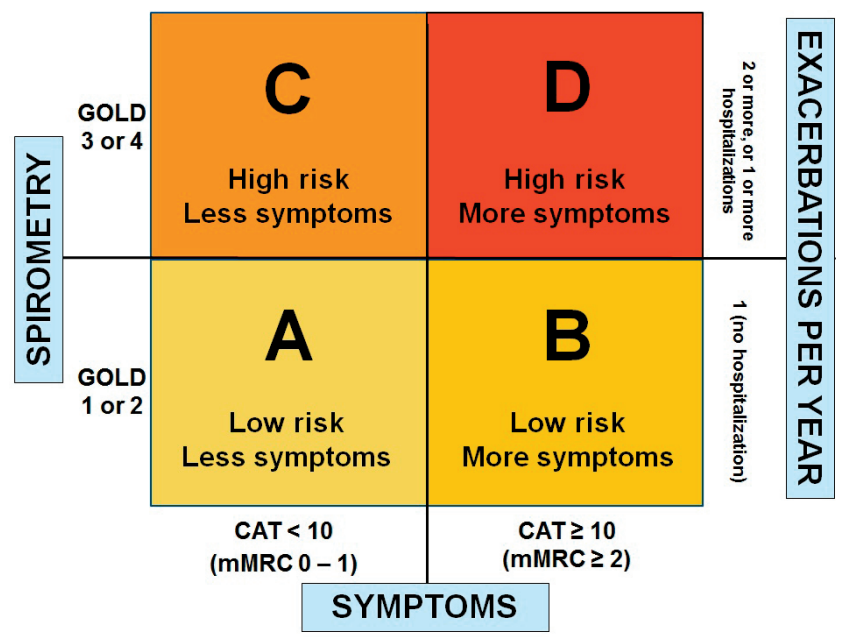

Figure 1: COPD patient classification
Inhaled bronchodilators are the mainstay of treatment in all patients with COPD as they provide symptom relief and reduce exacerbations. These are delivered via an aerosol or dry powder inhaler device. Long-acting bronchodilators are preferred to their short-acting counterparts. The latter should be used only as relievers on an as-needed basis. Long-acting $\beta_{2}$-agonists (LABA) currently available in South Africa are salmeterol, formoterol and indacaterol. The long-acting antimuscarinic agents (LAMA) available are tiotropium and glycopyrronium. COPD patients require either a $L A B A$ or $L A M A$ as regular maintenance therapy, but in more symptomatic patients, combinations of LABA and LAMA may be justified as the bronchodilator effects have been documented to be additive.

Inhaled corticosteroids are discouraged for COPD patients (unlike in asthma, where these drugs are obligatory), except in those experiencing frequent exacerbations, whose $\mathrm{FEV}_{1}$ is less than $60 \%$ predicted in the stable state, or those with features of asthma (ACOS). Systemic corticosteroids are indicated only as short course therapy during an exacerbation (Prednisone $40 \mathrm{mg}$ daily for 5 days). ${ }^{8}$ In this setting, they shorten duration of hospital admission and hasten improvement in lung function.

Theophylline, an oral methylxanthine, has only modest bronchodilator effect. Its disadvantage is its narrow therapeutic ratio and potential for severe adverse effects in toxic doses, such as atrial and ventricular arrhythmias, and generalized seizures. In low dose, it has been shown to reduce exacerbations. This drug has lost favour in view of its potential for significant sideeffects and the ready availability and greater efficacy of inhaled bronchodilators. However, in low income countries, it remains a therapeutic option.

The only phosphodiesterase-4 inhibitor available on the market is roflumilast. This is an oral drug recommended for patients with a chronic bronchitis phenotype who have frequent exacerbations and $\mathrm{FEV}_{1}<50 \%$ predicted. Side-effects such as nausea and diarrhoea are common, limiting its use.

A vital aspect of management often disregarded or overlooked is that of patient (and doctor) education in inhaler technique. In one of many studies demonstrating similar findings, misuse of inhaler devices was found to be extremely common amongst patients with both COPD and asthma ( $86 \%$ with metered dose inhalers and $71 \%$ with dry powder inhalers). ${ }^{9}$ However, it was reassuring that all patients studied were able to master the correct technique with appropriate training. Properly performed inhaler technique, particularly since there is a large variety of different inhaler devices available on the market, is crucial to effective treatment of the COPD patient.

Rehabilitation programmes have shown benefit in many aspects, e.g. improved exercise capacity, reduction in hospital admissions, and enhanced psychological wellbeing. Attention to nutritional support is also important, especially in patients with a low body mass index. Long-term domiciliary oxygen therapy is recommended for those with $\mathrm{PaO}_{2}<55 \mathrm{~mm} \mathrm{Hg}$ or $\mathrm{SaO}_{2}<88 \%$, or $\mathrm{PaO}_{2} 55-60 \mathrm{~mm} \mathrm{Hg}$ if there is also evidence of pulmonary hypertension, right heart failure or haematocrit $>55 \%$. Lung volume reduction procedures (surgically by lung resection and 
more recently, bronchoscopically, utilising coils or unidirectional valves) have a place in selected patients. Lung transplantation remains an option in well-resourced countries.

All COPD patients should receive annual influenza vaccine. Those older than 50 years or with other significant comorbid illness, e.g. cardiac disease, should also receive pneumococcal vaccine.

Antibiotics should be prescribed only for exacerbations caused by bacterial infection. Increased purulence of sputum is a helpful marker distinguishing bacterial from viral infections.

A common misconception in the management of COPD patients is that providing supplemental oxygen to these patients "removes their hypoxic drive" and results in $\mathrm{CO}_{2}$ retention. When high concentrations of oxygen are administered to some patients, $\mathrm{PaCO}_{2}$ does rise, sometimes to dangerously high levels. However, this worsening hypercapnia has been shown to be due to the following mechanisms in order of importance: worsened ventilation-perfusion matching due to the release of hypoxic pulmonary vasoconstriction, reduced affinity of haemoglobin for $\mathrm{CO}_{2}$ (Haldane effect) and decreased minute ventilation. ${ }^{10}$

The use of cardioselective $\boldsymbol{\beta}$-blockers for ischaemic heart disease in COPD patients is not contraindicated. Rather, they have been shown to reduce mortality in patients with coexistent coronary artery disease, and they may even reduce COPD exacerbations. ${ }^{11,12}$ These drugs should not be withheld if indicated for concurrent cardiovascular disease.

\section{Smoking cessation}

In a study by Zaballos et al.,13 $85 \%$ of anaesthesiologists enquired about a smoking history, but only 31\% informed patients about the health hazards related to smoking. In addition, only $23 \%$ advised patients to quit prior to elective surgery, and a significant minority (3\%) actually provided assistance to patients to help them quit.

It is the responsibility of all clinicians involved in the care of patients who smoke to promote smoking cessation. This should form a routine part of the preoperative evaluation by the anaesthesiologist. Methods for this important intervention include counselling, cognitive therapy to teach adjustment skills, and pharmacotherapy. Even brief counselling (as short as 3 minutes) has been demonstrated to increase the quit rate. Pharmacological agents which have proven value include nicotine replacement therapy, bupropion and varenicline. Clonidine and nortriptyline have also been shown to be helpful.

It is a fallacy that cessation of smoking less than eight weeks preoperatively increases the risk of postoperative pulmonary complications. ${ }^{14}$ This misconception has, unfortunately, persisted in the medical literature, including many textbooks and guideline documents. All smokers should be advised and encouraged to quit. Obviously the benefits of smoking cessation are greater the longer the period prior to anaesthesia and surgery, but this should not deter the initiation of smoking cessation strategies at the earliest opportunity. ${ }^{15}$
A detailed approach to smoking cessation is beyond the scope of this article, but readers are encouraged to peruse the clinical practice guideline published in 2013 by the South African Thoracic Society. ${ }^{16}$

\section{Asthma-COPD Overlap Syndrome (ACOS) ${ }^{17}$}

The recognition of patients with features of both asthma and COPD have led to the development of the term "asthma-COPD overlap syndrome". There remains controversy as to whether this is a distinct disease entity, whether these patients represent different phenotypes within each disease, or whether these patients have two separate diseases concurrently. The evolution of this concept stems from the finding that these patients have an unfavourable clinical course, with frequent exacerbations and disproportionate use of healthcare resources. Their outcome is worse than those suffering from either disease alone. In addition, appropriate treatment of these patients is uncertain as all clinical trials of asthma and COPD exclude this group. Currently there is no satisfactory definition of ACOS, suffice to say that such patients have several features of both asthma and COPD, and therefore do not fall clearly into one disease category or the other. These patients are generally over the age of 40 years.

From a practical and therapeutic perspective, a major reason for recognizing ACOS is the selection of drug treatment options. Essentially, the main impact is on the prescription of (a) maintenance inhaled corticosteroids, which should be limited to those patients who have enough features of asthma to justify their use (e.g. younger age of onset, greater variability of symptoms, identifiable triggers for symptoms, reversible airflow obstruction) and (b) maintenance long-acting bronchodilators, the mainstay of COPD drug therapy (rather than as-needed short-acting bronchodilators).

\section{Conclusion}

COPD is a heterogeneous chronic inflammatory disease affecting the airway and lung. It is common and an important cause of morbidity and mortality worldwide. Identification and elimination of risk factors such as tobacco smoking, render this disease potentially preventable.

\section{References}

1. Global Strategy for Diagnosis, Management, and Prevention of COPD (updated 2016). Global Initiative for Chronic Obstructive Lung Disease (COPD) 2016. http://www.goldcopd.org/guidelines-global-strategy-for-diagnosismanagement.html.

2. Chronic obstructive pulmonary disease. http://www.who.int/respiratory/copd/en/.

3. Tan WC, $\operatorname{Sin}$ DD, Bourbeau J, et al. Characteristics of COPD in never-smokers and ever-smokers in the general population: results from the CanCOLD study. Thorax. 2015;70(9):822-829.

4. COPD Assessment Test (CAT). http://www.thoracic.org/members/assemblies/ assemblies/srn/questionaires/copd.php.

5. Piquet J, Chavaillon JM, David P, et al. High-risk patients following hospitalisation for an acute exacerbation of COPD. Eur Respir J. 2013;42(4):946-955.

6. Hurst JR, Vestbo J, Anzueto A, et al. Susceptibility to exacerbation in chronic obstructive pulmonary disease. N Engl J Med. 2010;363(12):1128-1138.

7. Decramer M, Janssens W. Chronic obstructive pulmonary disease and comorbidities. The Lancet. Respiratory medicine. 2013;1(1):73-83.

8. Leuppi JD, Schuetz $P$, Bingisser $R$, et al. Short-term vs conventional glucocorticoid therapy in acute exacerbations of chronic obstructive pulmonary disease: the REDUCE randomized clinical trial. JAMA. 2013;309(21):2223-2231. 
9. Press VG, Arora VM, Shah LM, et al. Misuse of respiratory inhalers in hospitalized patients with asthma or COPD. J Gen Intern Med. 2011;26(6):635-642.

10. Aubier M, Murciano D, Milic-Emili J, et al. Effects of the administration of 02 on ventilation and blood gases in patients with chronic obstructive pulmonary disease during acute respiratory failure. The American review of respiratory disease. 1980;122(5):747-754.

11. Cazzola M, Matera MG. Beta-blockers are safe in patients with chronic obstructive pulmonary disease, but only with caution. Am J Respir Crit Care Med. 2008;178(7):661-662.

12. Du Q, Sun Y, Ding N, Lu L, Chen Y. Beta-blockers reduced the risk of mortality and exacerbation in patients with COPD: a meta-analysis of observational studies. PLoS One. 2014;9(11):e113048.
13. Zaballos M, Canal MI, Martinez R, et al. Preoperative smoking cessation counseling activities of anesthesiologists: a cross-sectional study. BMC Anesthesiol. 2015;15:60.

14. Shi Y, Warner DO. Brief preoperative smoking abstinence: is there a dilemma? Anesthesia and analgesia. 2011;113(6):1348-1351.

15. Wong J, Lam DP, Abrishami A, Chan MT, Chung F. Short-term preoperative smoking cessation and postoperative complications: a systematic review and meta-analysis. Can J Anaesth. 2012;59(3):268-279.

16. van Zyl-Smit RN, Allwood B, Stickells D, et al. South African tobacco smoking cessation clinical practice guideline. S Afr Med J. 2013;103(11):869-876.

17. Bateman ED, Reddel HK, van Zyl-Smit RN, Agusti A. The asthma-COPD overlap syndrome: towards a revised taxonomy of chronic airways diseases? The Lancet. Respiratory medicine. 2015;3(9):719-728. 